\title{
Recombinant Human T-Cell Leukemia Virus Types 1 and 2 Tax Proteins Induce High Levels of CC-Chemokines and Downregulate CCR5 in Human Peripheral Blood Mononuclear Cells
}

\author{
Christy S. Barrios, ${ }^{1,2}$ Muna Abuerreish,, Michael D. Lairmore, ${ }^{3}$ Laura Castillo,, \\ Chou-Zen Giam, ${ }^{4}$ and Mark A. Beilke ${ }^{1,2}$
}

\begin{abstract}
Human T-cell leukemia viruses types 1 (HTLV-1) and 2 (HTLV-2) produce key transcriptional regulatory gene products, known as Tax1 and Tax2, respectively. Tax1 and Tax2 transactivate multiple host genes involved in cellular immune responses within the cellular microenvironment, including induction of genes encoding expression of CC-chemokines. It is speculated that HTLV Tax proteins may act as immune modulators. In this study, recombinant Tax1 and Tax2 proteins were tested for their effects on the viability of cultured peripheral blood mononuclear cells (PBMCs), and their ability to induce expression of CC-chemokines and to downregulate the level of CCR5 expression in PBMCs. PBMCs obtained from uninfected donors were cultured in a range of Tax 1 and Tax 2 concentrations (10-100 pM), and supernatant fluids were harvested at multiple time points for quantitative determinations of MIP-1 $\alpha$ /CCL3, MIP-1 $\beta$ /CCL4, and RANTES/CCL5. Treatment of PBMCs with Tax1 and Tax2 proteins $(100 \mathrm{pM})$ resulted in a significant increase in viability over a 7 -d period compared to controls $(p<0.01)$. Both Tax1 and Tax2 induced high levels of all three CC-chemokines over the dosing range compared to mock-treated controls $(p<0.05)$. The gated population of lymphocytes treated with Tax2, as well as lymphocytes from HTLV-2-infected donors, showed a significantly lower percentage of CCR5-positive cells compared to those of uninfected donors and from mock-treated lymphocytes, respectively $(p<0.05)$. These results suggest that Tax1 and Tax2 could promote innate immunity in the extracellular environment during HTLV-1 and HTLV-2 infections via CC-chemokine ligands and receptors.
\end{abstract}

\section{Introduction}

$\mathbf{H}$ uman T-Cell leukemia ViRus types 1 (HTLV-1) AND 2 (HTLV-2) are the first described human retroviruses. HTLV-1 and HTLV-2 have similar biological properties, tropism for $\mathrm{T}$ lymphocytes, and mechanisms of transmission $(17,18)$. HTLV-1 is the causative agent of adult T-cell leukemia/lymphoma (ATLL) $(21,41,55)$, and a progressive neurodegenerative disease known as tropical spastic paraparesis / HTLV-1-associated myelopathy (TSP/HAM) $(37,43)$. HTLV-1 has also been implicated as a cause of other virally-induced autoimmune and inflammatory syndromes (48). In contrast, HTLV-2 infections are generally asymptomatic and may induce subtle but important effects on the host immune system.
HTLV-2-infected blood donors exhibit persistently increased absolute lymphocyte and platelet counts (4). High rates of human immunodeficiency virus-1 (HIV-1)/HTLV co-infections exist in HTLV-1-endemic areas, and in metropolitan areas where HTLV-2 is transmitted through needle sharing. Among persons with HIV-1 and HTLV-2 co-infections, retroviral co-infections have been shown in both U.S. and European studies to result in altered clinical outcomes, slower rates of $\mathrm{CD}^{+}{ }^{+} \mathrm{T}$-cell decline, and/or delayed progression to AIDS $(6,51)$.

These observations have prompted our group to investigate the possibility that upregulated expression of HTLV-1 and HTLV-2 transcriptional activating proteins, known as Tax1 and Tax2, might play key roles in modulating innate

\footnotetext{
${ }^{1}$ Infectious Diseases Division, Department of Medicine, Medical College of Wisconsin, Milwaukee, Wisconsin.

${ }^{2}$ Research Service 151-I, Clement J. Zablocki Veterans Affairs Medical Center, Milwaukee, Wisconsin.

${ }^{3}$ Department of Veterinary Biosciences, The Ohio State University, Columbus, Ohio.

${ }^{4}$ Department of Microbiology and Immunology, Uniformed Services University of the Health Sciences, Bethesda, Maryland.
} 
immunity during HIV/HTLV co-infection. Tax1 and Tax2 are also able to activate cellular genes via the CREB and NF$\kappa \mathrm{B}$ pathways, resulting in upregulated expression of cytokines, chemokines, and other cellular gene products in the cellular microenvironment $(3,8,26,44,49)$.

Chemokines are small structurally-related molecules belonging to a large supergene family of 8 - to $14-\mathrm{kDa}$ basic heparin-binding proteins, and have been identified to play an important role in innate immunity by inducing the directional migration of a number of inflammatory cells, and activating specific leukocyte populations (30). The approximately 50 human chemokines segregate into four subfamilies based upon their unique sequence homology and the position of cysteine residues in the proteins. The largest family consists of CC-chemokines (or $\beta$-chemokines), and are so named because they have two adjacent cysteines near their amino terminus. This group includes at least 28 members named CC-chemokine ligands (CCL) 1 to 28 . CC-chemokines attract mononuclear cells (monocytes, dendritic cells, eosinophils and NK cells) to sites of chronic inflammation. The most thoroughly characterized CC-chemokine is monocyte chemoattractant protein-1 (MCP-1 or CCL2), which induces monocytes to leave the bloodstream and enter the surrounding tissue to become tissue macrophages. Other CC-chemokines include macrophage inflammatory protein (MIP)- $1 \alpha$ (CCL3), MIP-1 $\beta$ (CCL4), and RANTES (CCL5). CCL5 attracts cells such as $\mathrm{T}$ cells, monocytes (expressing CCR5), eosinophils, and basophils (expressing CCR1 and/ or CCR3 receptors) (30). Proteins and receptors of the CCchemokine subfamily play a key role in modulating HIV-1 infection and replication $(1,11,12)$. In this study we investigated whether Tax2 may induce the production of CCchemokines (MIP-1 $\alpha$, MIP-1 $\beta$, and RANTES), and result in the binding and consequently modulation of CCR5, the coreceptor for the R5 tropic strain of HIV-1.

Previous work has emphasized the effects of HTLV-1 and Tax1 on host gene responses, given the ability of HTLV-1 to cause significant hematologic and neurologic disease. Lesser attention has been directed to the effects of HTLV-2 on host immunity. Given the high prevalence of HTLV-2 in certain populations, this work is clinically relevant. In this research study, it was shown that recombinant Tax2 (Tax2) is a potent inducer of CC-chemokines in peripheral blood mononuclear cells (PBMCs). Additionally, it was demonstrated that Tax2 downregulated the expression of CCR5 on lymphocytes. This line of experimental research may be significant to clinical investigations of HIV/HTLV co-infections $(6,51)$.

\section{Material and Methods}

\section{Protein purification}

Recombinant Tax1 and Tax2 proteins were expressed in E. coli and purified using an adapted method (27). The BL21(DE3) strain of E. coli, containing the pET-Tax1 or the pET-Tax2 expression vectors, were generated as previously described (50). The bacteria were grown in Luria broth base ( $25 \mathrm{~g} / \mathrm{L}$; Invitrogen, Carlsbad, CA) at $18^{\circ} \mathrm{C}$ under ampicillin (Sigma-Aldrich, St. Louis, MO) selection in the presence of $0.1 \mathrm{mM}$ isopropyl $\beta{ }^{-}{ }_{\mathrm{D}}$-thiogalacto-pyranoside (IPTG) (SigmaAldrich). Extracellular bacterial extract (ebe) without the Tax vectors was used as a background mock control. Determination of protein concentrations was performed using a BCA protein assay (Pierce Protein Research Products, Rockford, IL), according to the manufacturer's specifications. Proteins were analyzed for the presence of endotoxin at the concentrations used in the experiments, using the Limulus amebocyte lysate (LAL) test (E-TOXATE; Sigma-Aldrich). To reduce contamination of endotoxin in the purified proteins, all glassware was autoclaved for $1 \mathrm{~h}$, then heated in an oven at $175^{\circ} \mathrm{C}$ for $3 \mathrm{~h}$. Solution transfers were performed by endotoxin-free devices.

The purity and molecular weights of the recombinant proteins were determined by SDS-PAGE analysis. The fractionated proteins were electrophoretically transferred to nitrocellulose membranes and analyzed by Western blotting. The membranes were incubated with a rabbit polyclonal antibody against two synthetic oligopeptides, the first representing a conserved central domain of Tax1 and Tax2 proteins (amino acid residues 74-84), and the second representing the C-terminal domain (amino acids 320-331) of the Tax2 sequence (1:500, serum GP3738, a generous gift of Dr. Renaud Mahieux, Pasteur Institute, Paris, France) (31). After extensive washing the membranes were incubated with a biotinylated goat anti-rabbit IgG (1:1000; SigmaAldrich), followed by alkaline phosphatase (AP)-streptavidin (1:30,000; Zymed Laboratories, Carlsbad, CA), and developed by an AP conjugate substrate kit (Bio-Rad Laboratories, Inc., Hercules, CA).

\section{Transfection and luciferase reporter gene analysis}

To determine whether the recombinant Tax proteins retained the ability to initiate viral transcription, an HTLV-1 pLTR-Luc reporter assay was employed. HTLV-pLTR contains three repeats of the Tax-responsive element (TRE), and was used for nucleofection experiments as follows (36). First the assay was optimized via nucleofection of HTLV pLTRLuc into transformed HTLV-2, producing T-cell line MoT and the HTLV-1-producing T-cell line MT2, which are known to contain high levels of Tax2 and Tax1, respectively. Cell lines were nucleofected with $2.9 \mu \mathrm{g}$ of HTLV pLTR-Luc, according to the manufacturer's instructions under conditions for T cells (Amaxa, Gaithersburg, MD). Following optimization, Jurkat cells were incubated with $100 \mathrm{pM}$ of recombinant Tax proteins prior to and during nucleofection. Two micrograms of maxGFP plasmid was used in each experiment as a control for transfection efficiency, and GFPexpressing Jurkat cells were analyzed by fluorescence microscopy. Cells were harvested $18 \mathrm{~h}$ after transfection, luciferase activity was measured with a luciferase assay kit (Promega Corp., Madison, WI), and luciferase light units were measured with the Synergy 2 luminescence microplate reader (BioTek Instruments, Inc., Winooski, VT). The measured light units were normalized by total protein content by dividing luciferase values by protein concentration to obtain normalized relative luciferase units (RLU). The effect of Tax transactivation was also expressed as fold activation compared with the basal activity of LTR-Luc without Tax $(10,22)$. Representative data of triplicate experiments is shown as mean \pm SEM.

\section{Processing PBMC samples}

Blood samples from three healthy donors were obtained following informed consent using a protocol that was 
approved by the Institutional Review Board for Human Investigation of the Milwaukee Veterans Affairs, Research Service Committee. Donors were confirmed to be HIV-1 and HTLV-1/-2 seronegative by ELISA assay. Whole blood was collected in CPT/Vacutainer BD tubes (BD Biosciences, San Jose, CA), and PBMCs were obtained following manufacturer recommendations. Cryopreserved PBMC aliquots from three HTLV-2-infected individuals were also tested for their ability to induce CC-chemokines and to express CCR5 receptor (a generous gift of Dr. Edward L. Murphy, University of California, San Francisco) (4).

\section{Uptake of recombinant Tax proteins into PBMCs and functional cellular assays (viability, chemokine determination, and CCR5 expression)}

In order to determine the rate and uptake efficiency of recombinant Tax proteins, PBMCs from a healthy donor were incubated in RPMI complete medium (supplemented with $10 \%$ FBS, 100 units $/ \mathrm{mL}$ penicillin, and $100 \mu \mathrm{g} / \mathrm{mL}$ streptomycin) for $40 \mathrm{~h}$ with $100 \mathrm{pM}$ of Tax proteins (Tax1 and Tax2) or ebe as a negative control. Cells were cytocentrifuged onto glass slides and fixed with $4 \%$ paraformaldehyde. Indirect immunofluorescence assay (IFA) was performed using serum anti-Tax2 (1:100 GP3738), and goat anti-mouse fluoresceinlabeled antibodies (1:200; KPL, Gaithersburg, MD). Normal rabbit serum was used as a background control. Cells were observed and photographed with a BX51 Olympus epifluorescence microscope (Tokyo, Japan), and images were captured using the DP software interface to the microscopy system.

Cell viability. To examine the effects of the recombinant proteins on cell viability, PBMCs from three healthy donors were co-cultured in vitro with $100 \mathrm{pM}$ of Tax1 protein, Tax2 protein, or ebe, in RPMI complete medium for 7 days. Cell viability was determined by trypan blue exclusion using light microscopy. Trypan blue has been found to be an ideal stain because it easily diffuses across cell membranes of dead or dying cells, but cannot cross membranes of live cells. This method is very simple and widely used for the determination of total numbers of viable cells in a cell suspension. Although time consuming, it uses a very small volume of cell culture to quantify viable cells (52). Ten microliters of cell suspension was placed in an Eppendorf tube with an equal volume of $0.4 \%$ trypan blue, gently mixed, and allowed to stand for $5 \mathrm{~min}$ at room temperature. The number of viable cells was counted, and the average number in each quadrant was calculated, and multiplied by $2 \times 10^{4}$ to determine numbers of cells $/ \mathrm{mL}$.

PBMC cultures and CC-chemokine determination in culture supernatants. PBMCs $\left(1 \times 10^{6} / \mathrm{mL}\right)$ were cultured in RPMI complete medium alone or with $100 \mathrm{pM}$ or $10 \mathrm{pM}$ of Tax2, Tax1, or ebe in 24-well plates. PBMCs treated with a mitogen (phytohemagglutinin, PHA; Sigma-Aldrich) were used as a positive control. Cell-free culture supernatants were collected at 24,48 , and $72 \mathrm{~h}$, and kept at $-20^{\circ} \mathrm{C}$ until use. The levels of MIP- $1 \alpha$, MIP- $1 \beta$, and RANTES in the supernatants were assayed by ELISA (DuoSet ELISA development kits; R\&D Systems, Inc., Minneapolis, MN), following the manufacturer's instructions. Absorbance val- ues were read at $450 \mathrm{~nm}$ in a microplate reader (BioTek Instruments). The concentrations (pg/mL) of chemokines were quantified with a standard curve using the manufacturer's software.

CCR5 expression by flow cytometry. In parallel experiments, treated PBMCs were collected for determinations of CCR5 expression by flow cytometry. Briefly, Tax-treated PBMCs were washed and blocked for non-specific FCRmediated binding. The cells were stained for CCR5 expression using phycoerythrin (PE)-labeled anti-CCR5 monoclonal antibody (mAb, clone 2D7, BD Bioscience), or PE mouseIg $_{\text {Ga }}$ kappa (clone G155-178; BD Bioscience) isotype control. The 2D7 mAb recognizes a conformation-dependent epitope in the second extracellular loop of CCR5, and was selected since this antibody has been shown to block ligand and gp120 binding, and is one of the most potent inhibitors of R5 virus cell entry (24). PBMCs from HTLV-2-infected and uninfected individuals were also stained using the same procedure. The cells were analyzed by flow cytometry using a LSR II Flow Cytometer (BD Biosciences). Data were analyzed using FlowJo software version 7.6/9.0 (Tree Star Inc., Ashland, OR).

\section{Statistical analysis}

Data are presented as mean \pm SEM. To determine significant differences between group averages, the data were analyzed with Excel and Minitab for Windows software using one-way ANOVA, followed by one-tailed Student's $t$-test assuming equal variances. $p$ Values less than 0.05 were considered to be statistically significant.

\section{Results}

\section{Western blot and endotoxin analysis of Tax1 and Tax2 recombinant proteins}

Western blot analysis of recombinant Tax1 and Tax2 proteins showed bands at 40 and $37 \mathrm{kDa}$, respectively (Fig. 1A). While positive controls containing 0.06 endotoxin U/ $\mathrm{mL}$ (derived from E. coli 0.55:B5 lipopolysaccharide) yielded the formation of a hard gel permitting complete inversion of the tube without disruption, the recombinant protein preparations were found to be endotoxin-free at the levels used for in vitro experimentation (1-1000 pM), as tested by this assay.

\section{Reporter gene analysis of recombinant Tax proteins with HTLV pLTR-LUC}

The main biological function of Tax1 and Tax2 proteins is the transcriptional transactivation of HTLV-LTR, leading to efficient expression of viral RNA and viral replication in the infected cell. Tax proteins have been involved in the transactivation of cell host genes. In order to determine whether recombinant Tax proteins were able to transactivate HTLV LTR promoter, reporter gene assays were performed using HTLV-pLTR-Luc as a reporter, and recombinant Tax proteins as effectors. Results of normalized RLU mean \pm SEM values were $10617 \pm 1196$ for Tax1, 11296 \pm 1033 for Tax2, $2274 \pm 120$ for ebe, and $1298 \pm 178$ for untreated Jurkat. Recombinant Tax1 and Tax2 transactivated the HTLV LTR 
A

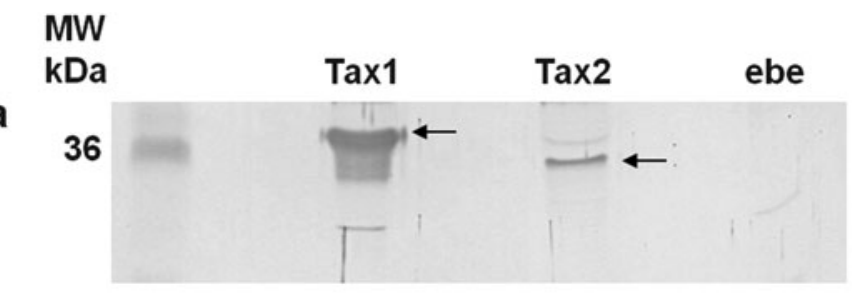

b

B
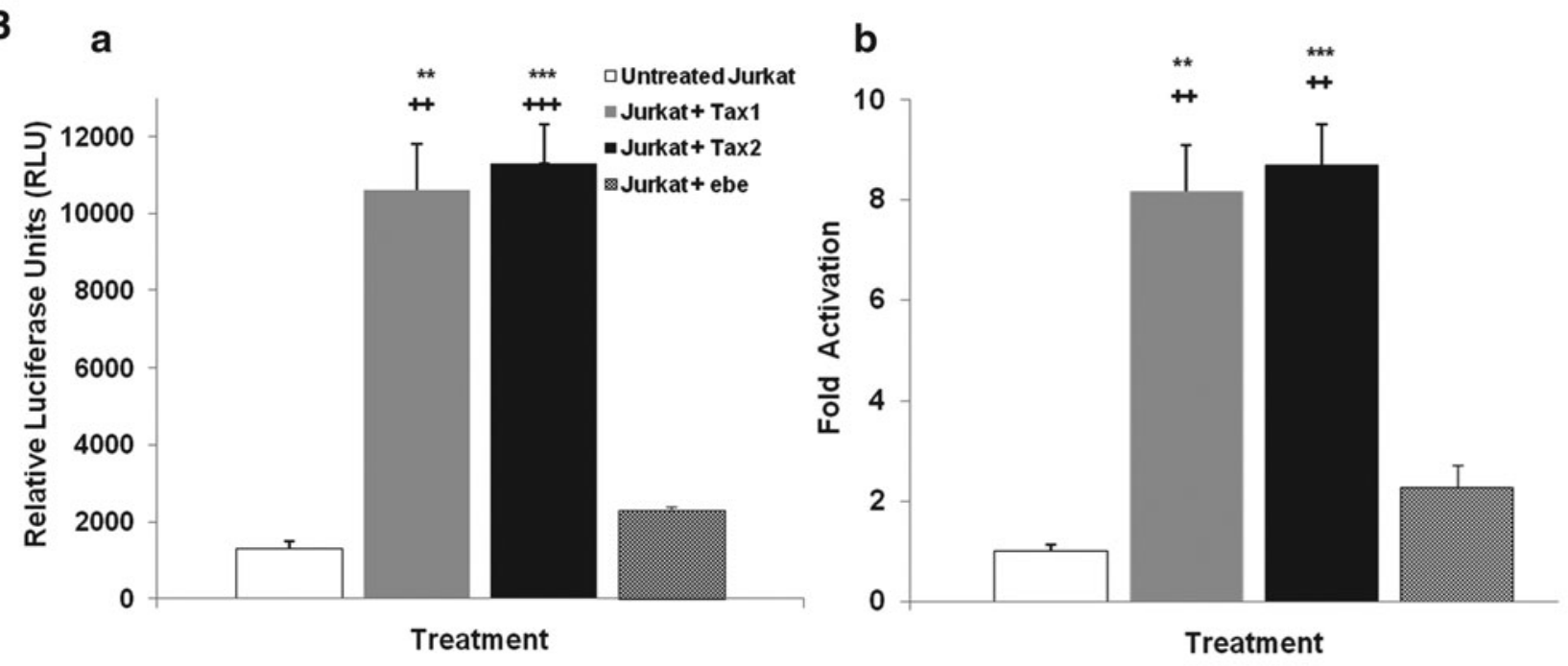

C
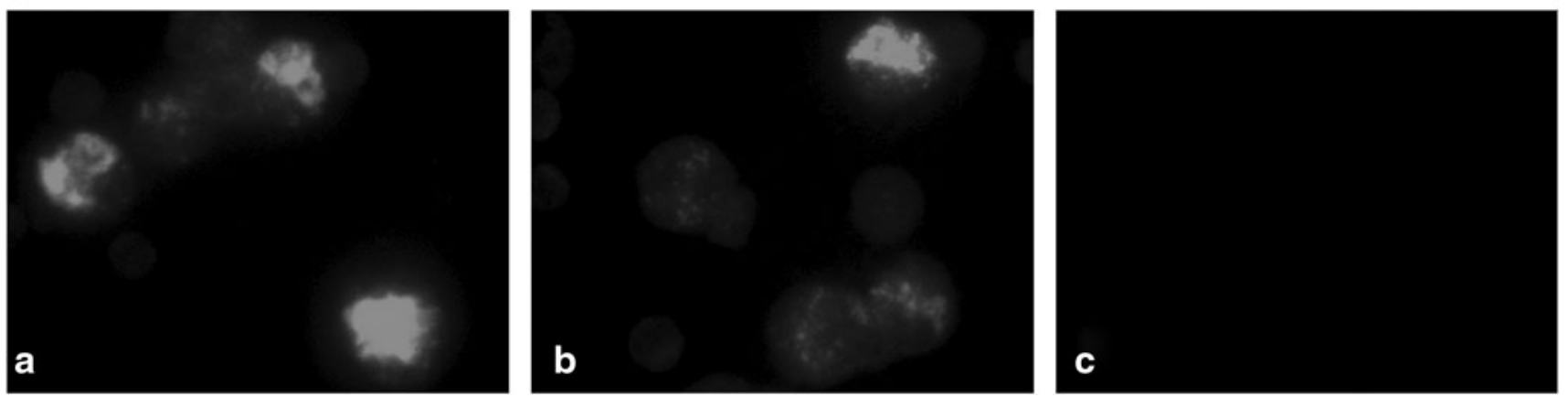

FIG. 1. Western Blot of purified Tax proteins, recombinant Tax transactivation of pHTLV LTR promoter activity, and uptake of recombinant Tax proteins by PBMCs. (A) Western blot of Tax proteins. Recombinant Tax1, Tax2, and extracellular bacteria extract (ebe) were loaded onto 10\% polyacrylamide gels for SDS-PAGE, and transferred to nitrocellulose membranes for Western blotting. The blot was probed with (a) anti-Tax antiserum or (b) normal rabbit serum, and detected using biotinlabeled antibodies, alkaline phosphatase-labeled streptavidin, and the chromogenic substrate BCIP/NBT. (B) Transactivation of HTLV LTR promoter by recombinant Tax. Transactivation was expressed as normalized RLU (a) and as fold activation (b). Fold activation of HTLV LTR promoter activity was determined as RLU of Tax-treated Jurkat transfected with HTLV pLTRLuc promoter, divided by the RLU of untreated Jurkat transfected with HTLV pLTR. Representative results of triplicate experiments are shown as the mean \pm SEM $\left({ }^{* *} p<0.01,{ }^{* * *} p<0.001\right.$ versus untreated Jurkat; $++p<0.01,+++p<0.001$ versus mock-treated). (C) Immunofluorescence of Tax-treated PMBCs. PBMCs treated with recombinant Tax1 (a), Tax2 (b), and ebe (c), after $40 \mathrm{~h}$ were cytocentrifuged, fixed with paraformaldehyde, and examined by indirect immunofluorescence using antiTax antiserum and goat anti-rabbit-FITC-labeled antibody. 
promoter activity 8.2- and 8.7-fold, respectively, over untreated Jurkat cells $(p<0.01$; Fig. 1B).

\section{Cell uptake of rTax1 and rTax2 and enhanced PBMC viability}

Efficient uptake of the recombinant Tax proteins by PBMCs was demonstrated by immunofluorescence analysis within 24-40 h of in vitro culture with the recombinant proteins (Fig. 1C). To establish any potential toxic effect of the recombinant proteins, cell viability was determined. Of interest, treatment with both recombinant Tax proteins resulted in a statistically significant increase $(p<0.05)$ in PBMC viability compared to controls over a 7-d assay period (Fig. 2).

\section{CC-chemokine production and concomitant CCR5 expression induced by Tax proteins}

CC-chemokines analyzed by ELISA in supernatants from in vitro cultured Tax-treated PBMCs. To determine whether Tax proteins could stimulate CC-chemokines, $10-100$ pM Tax1 and Tax 2 proteins were added to in vitro cultured PBMCs from healthy donors. Supernatants were collected at 24, 48, and $72 \mathrm{~h}$, and ELISA was performed for the determination of CC-chemokines. Maximal levels of MIP- $1 \alpha$ (30.9 \pm 13.3 , $39.6 \pm 21.7$, and $15.9 \pm 3.1 \mathrm{ng} / \mathrm{mL}$ ) were observed in PBMCs treated with $100 \mathrm{pM}$ Tax2, versus $0.2 \pm 0.2,0.06 \pm 0.05$, and $0.02 \pm 0.01 \mathrm{ng} / \mathrm{mL}$ for untreated PBMCs at 24,48 , and $72 \mathrm{~h}$, respectively (Fig. 3A). Significant levels of MIP- $1 \alpha$ were also demonstrated when PBMCs were treated with $10 \mathrm{pM}$ of Tax2 $(p<0.05$; Fig. 3B). MIP-1 $\beta$ was also detected in the supernatants of Tax2-treated (100 pM) PBMCs (25.7 $\pm 3.0,27.3 \pm 7.0$, and $20.6 \pm 3.3 \mathrm{ng} / \mathrm{mL}$ versus $2.8 \pm 1.1,1.4 \pm 0.6$, and $1.04 \pm 0.6 \mathrm{ng} / \mathrm{mL}$, at 24, 48, and $72 \mathrm{~h}$, respectively; Fig. 3C), and were significantly elevated when treated with $10 \mathrm{pM}$ of Tax2 at the three time points studied (Fig. 3D). Although there was spontaneous release of RANTES by untreated PBMCs, Tax2 induced higher levels of RANTES in Tax2-treated (100 pM) PBMCs (22.1 $\pm 4.5,21.9 \pm 4.2$, and $18.2 \pm 5.3 \mathrm{ng} / \mathrm{mL}$ versus $10.5 \pm 2.0,5.0 \pm 1.9$, and $3.2 \pm 1.4 \mathrm{ng} / \mathrm{mL}$, at 24,48 , and $72 \mathrm{~h}$, respectively) compared to controls (Fig. 3E). In addition, RANTES remained significantly upregulated when $10 \mathrm{pM}$ of Tax2 was used at 48 and $72 \mathrm{~h}$ compared to untreated controls (Fig. 3F). Tax1 protein at $100 \mathrm{pM}$ induced higher expression of MIP- $1 \alpha$, MIP- $1 \beta$, and RANTES by PBMCs compared to controls, at 24, 48, and $72 \mathrm{~h}$ (Fig. 3A, C, and E). PHA-stimulated PBMCs expressed high levels of MIP- $1 \alpha$, MIP- $1 \beta$, and RANTES (Fig. 3G, H, and I, respectively).

CCR5 expression by FACS in Tax-treated PBMCs. The high-level expression of CC-chemokines in PBMCs treated with Tax proteins suggested the possibility that CCR5 expression could consequently be altered in PBMCs treated with Tax2 proteins. In preliminary experiments, supernatants of in vitro cultured PBMCs from HTLV-2-infected individuals showed significantly high levels of RANTES at 72 and $144 \mathrm{~h}(p<0.05)$, compared to PBMCs from uninfected controls (Fig. 4A). PHA-stimulated PBMCs released high levels of RANTES (Fig. 4B). Since we used in vitro cultured PBMCs from HTLV-2 patients, we ensured virus replication and possible detection of CC-chemokines in these supernatants at extended intervals up to day 6 (144h). Lymphocytes from the HTLV-2-infected patients also showed a tendency toward a lower percentage of CCR5-PE-positive cells, reaching a significant difference at $72 \mathrm{~h}$ of in vitro culture compared to lymphocytes from normal donors $(p<0.05$; Fig. $4 C)$. A possible explanation for the discrepancy in the maximal expression of CC-chemokines observed in Figs. 3 and 4 is that recombinant Tax protein was added to the in vitro cultured PBMCs, and Tax could immediately elicit the production of CC-chemokines by the mononuclear cells (Fig. 3), while HTLV-2-infected PBMCs required time for tax gene expression to occur (Fig. 4). Of interest, once RANTES was

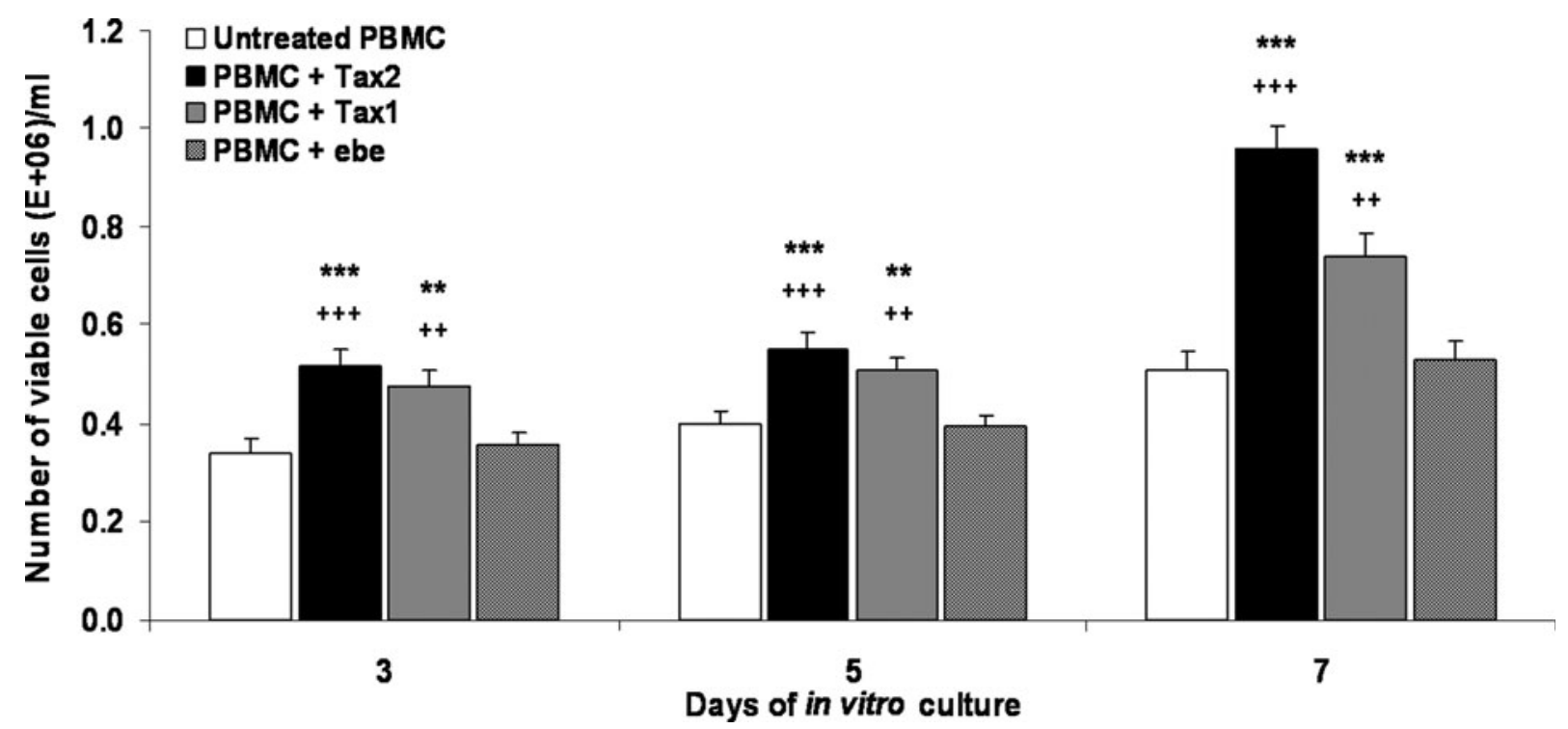

FIG. 2. Viability of PBMCs co-cultured with Tax proteins. The number of viable cells was determined on days 3, 5, and 7 of in vitro culture with $100 \mathrm{pM}$ recombinant Tax1 or Tax2 proteins. Extracellular bacterial extract (ebe) and untreated PBMCs were used as background controls. Numbers of viable cells were determined by counting using the trypan blue exclusion method. Data represent the mean of three independent experiments including three healthy PBMCs donors. Data shown are the mean values \pm SEM $\left({ }^{* *} p<0.01,{ }^{* * *} \mathrm{p}<0.001\right.$ versus untreated PBMCs; $++p<0.01,+++p<0.001$ versus ebe). 

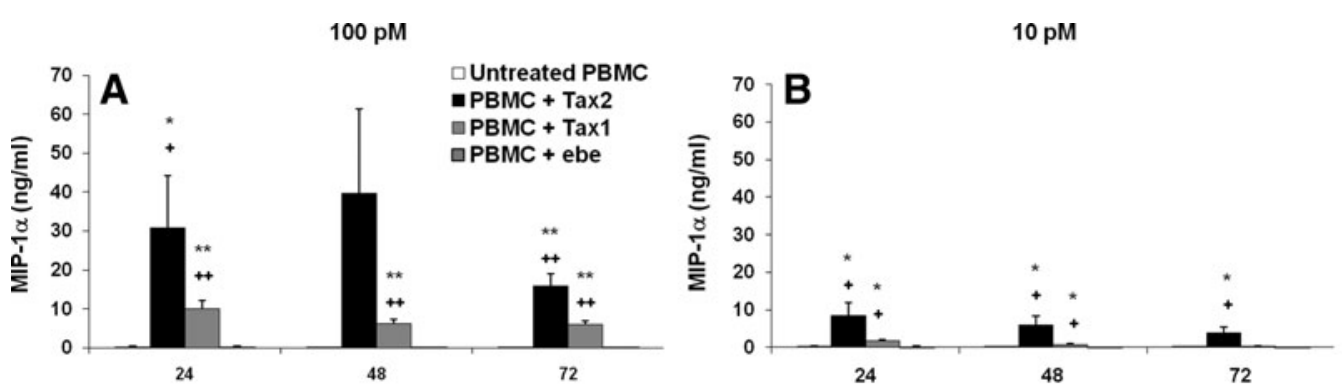

PHA stimulated-PBMC
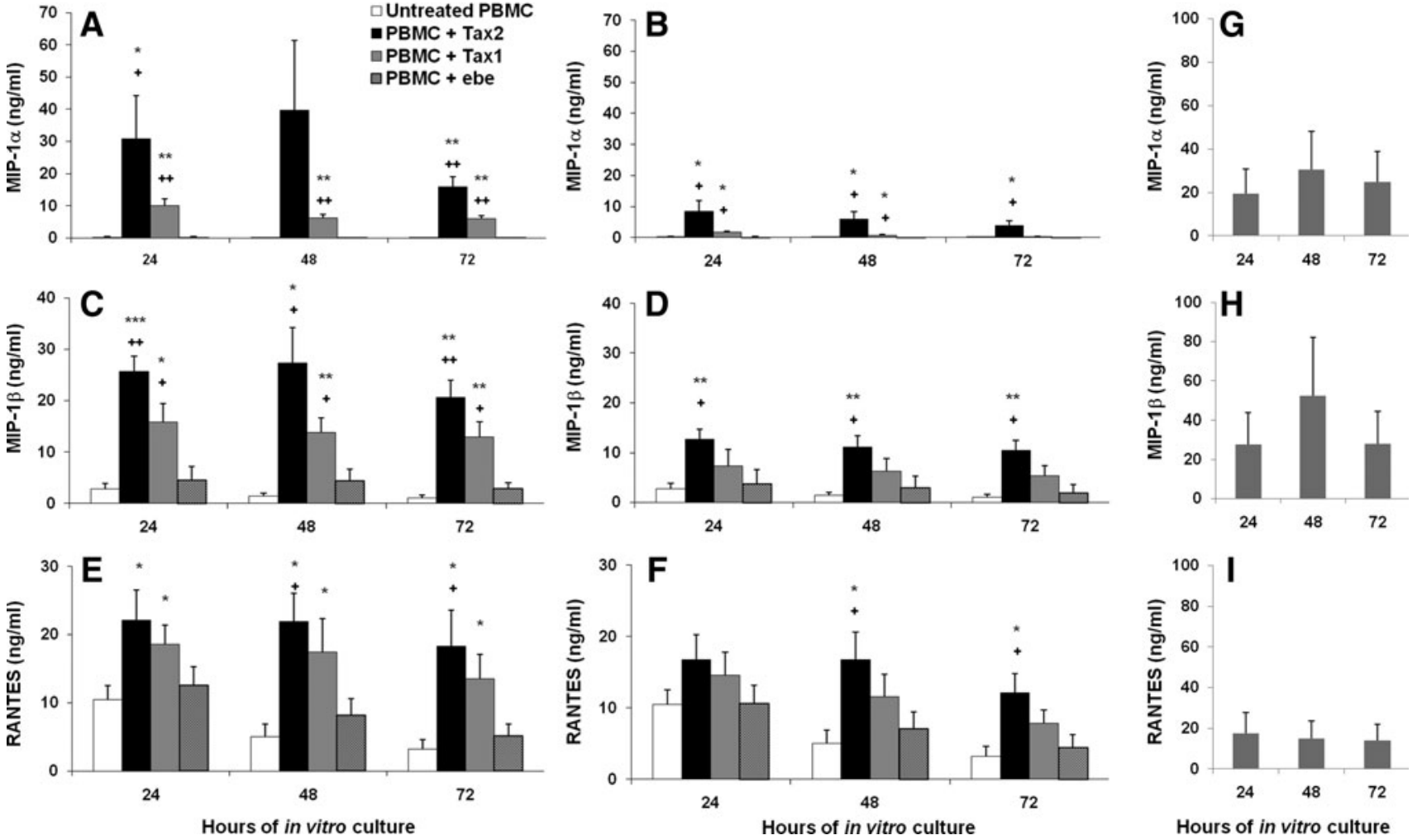

FIG. 3. CC-chemokine levels of PBMCs treated with Tax proteins. Tax proteins at $100 \mathrm{pM}(\mathbf{A}, \mathbf{C}$, and E), or $10 \mathrm{pM}(\mathbf{B}, \mathbf{D}$, and F) induced production of MIP-1 $\alpha,(\mathbf{A}$ and $\mathbf{B}), \mathrm{MIP}-1 \beta,(\mathbf{C}$ and $\mathbf{D})$, and RANTES (E and F) in PBMC cultures from three HIV-1and HTLV-1/2-seronegative donors. PHA-stimulated PBMCs $(5 \mu \mathrm{g} / \mathrm{mL}$, positive control) released MIP- $1 \alpha(\mathrm{G}), \mathrm{MIP}-1 \beta(\mathbf{H})$, and RANTES (I). Untreated PBMCs were used as a background control. Results are expressed as mean and SEM of three sets of PBMCs from HIV-1/HTLV-seronegative donors $\left({ }^{*} p<0.05,{ }^{* *} p<0.01\right.$, and ${ }^{* * *} p<0.001$ versus untreated; $+p<0.05$ and $++p<0.01$ versus ebe).

detected in the supernatants of cultured HTLV-2-infected PBMCs at $72 \mathrm{~h}$, and the percentage of CCR5-positive cells at that time point was also significantly downregulated (Fig. 4). Hence, PBMCs were cultured in the presence of recombinant Tax proteins and controls, and after $48 \mathrm{~h}$ the cells were assayed for levels of CCR5 expression by flow cytometry. Lower percentages of CCR5-positive cells were expressed in lymphocytes from Tax2- and Tax1-treated PBMCs $(10.0 \%$ and $17.4 \%$ at $100 \mathrm{pM}$, and $9.3 \%$ and $20.3 \%$ for $10 \mathrm{pM}$, respectively), compared to ebe-treated $(45.0 \%$ for $100 \mathrm{pM}$ and $42.7 \%$ for $10 \mathrm{pM})$, or untreated PBMCs (35.0\%; Fig. 5A). In separate experiments, PBMCs from three healthy donors were treated with Tax proteins for 24, 48, and $72 \mathrm{~h}$. Results indicated that a Tax2-induced reduction of CCR5 expression was already observed at the 24-h time point, and continued at 48 and $72 \mathrm{~h}$, compared to untreated PBMCs and those treated with ebe (Fig. 5B). Tax2 showed significant downregulation of the CCR5 receptor at all concentrations tested $(p<0.05)$. Tax1 at 100 and $10 \mathrm{pM}$ showed significant CCR5 downregulation, mainly at $24 \mathrm{~h}(p<0.05$; Fig. 5B).

\section{Discussion}

In this study, recombinant HTLV-1 and HTLV-2 Tax proteins were shown to induce high levels of CC-chemokine production by human PBMCs, with concomitant down- regulation of CCR5 receptor expression. Treatment with the recombinant proteins was also associated with a statistically significant improvement in PBMC viability over a 7-d assay period. These data suggest the potential involvement of Tax1 and Tax 2 in the maintenance of cell pool numbers, possibly due to inhibition of apoptosis and/or induction of cell cycle promotion/proliferation $(8,47)$. Furthermore, it was demonstrated that the recombinant proteins were efficiently taken up by PBMCs, and that the proteins induced the transactivation of HTLV LTR in transfected Jurkat cells, suggesting that they retained the function of the native virally-derived Tax proteins. Kamoi et al. (22) reported a 20-fold increase of Tax1 transactivation in HTLV pLRT-Luc-transfected Jurkat cells, yet Tax was expressed via tax gene co-transfection rather than treated with Tax proteins.

Tax1 is centrally involved in the complex steps of leukemogenesis following years of subclinical HTLV-1 infection. Tax1 functions in a pleiotropic manner, including constitutive activation of transcription factors, modification of signal transduction pathways, alteration of tumor suppressor protein functions, modulation of cell-cycle checkpoint proteins, interference with DNA repair, and inhibition of apoptosis. Tax1 has been implicated as the transforming protein of HTLV-1, and is centrally involved in the complex steps of leukemogenesis following years of subclinical infection. Tax1 is a potent activator of the NF- $\kappa \mathrm{B} /$ Rel family of transcription 

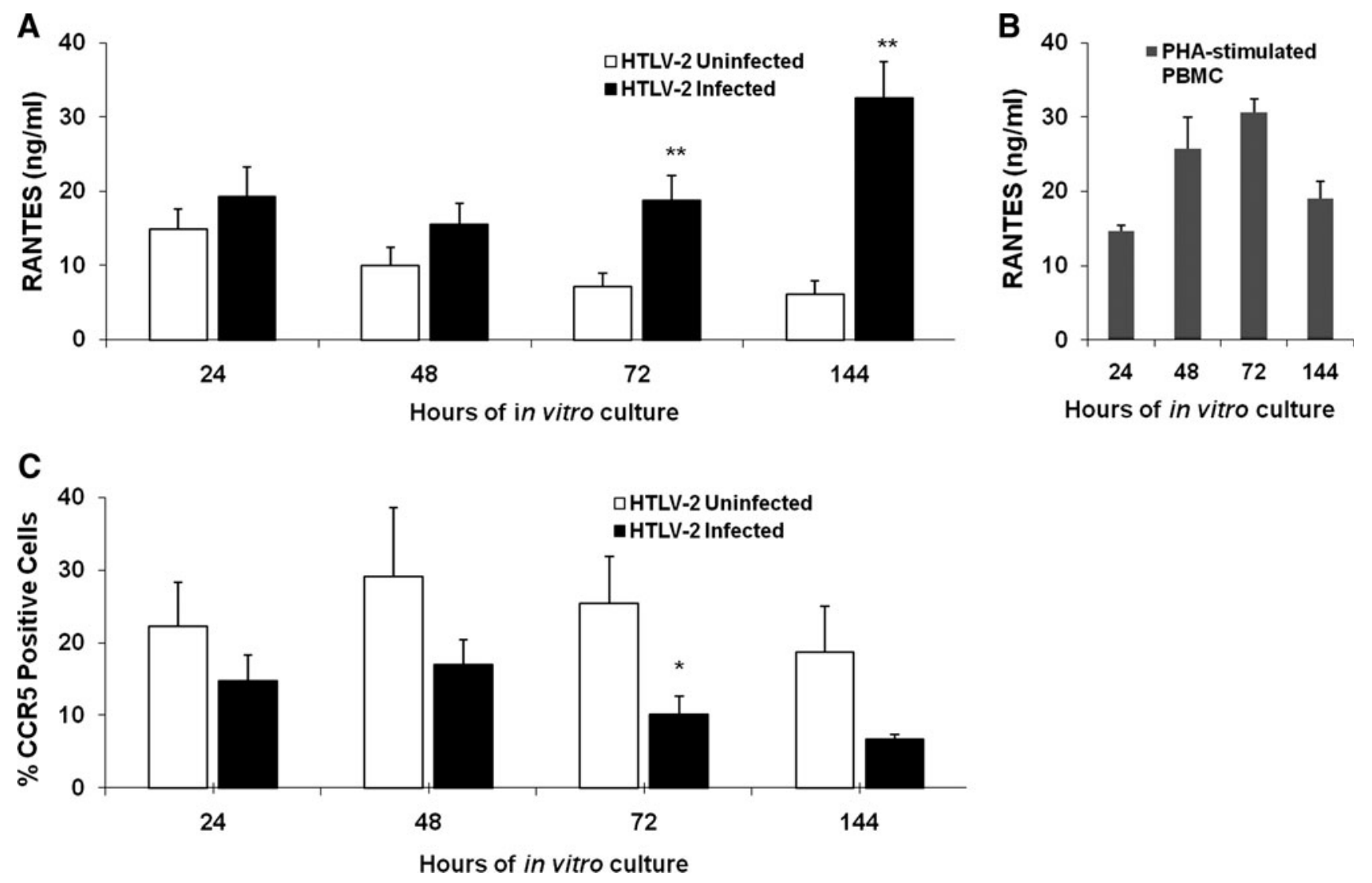

FIG. 4. CCR5 receptor expression and RANTES production from in vitro cultured PBMCs of HTLV-2-infected and uninfected individuals. (A) Supernatants collected after 24, 48, 72, and $144 \mathrm{~h}$ from the in vitro cultured cells from HTLV-2-infected and uninfected individuals were tested for CCL5/RANTES expression by ELISA. (B) Culture supernatants of PBMCs stimulated with PHA $(5 \mu \mathrm{g} / \mathrm{mL})$ were used as positive control. (C) PBMCs from infected and uninfected individuals were in vitro cultured for 24, 48, 72, and $144 \mathrm{~h}$, and stained with CCR5-PE antibody and analyzed by FACS. Results are presented as mean and SEM of percentages of CCR5-PE-positive cells $\left({ }^{*} p<0.05,{ }^{* *} p<0.01\right.$ versus uninfected samples).

factors by activating I- $\kappa$ B kinase (IKK), the Ser/Thr kinase that targets the destruction of I- $\kappa$ Bs $(19,23)$. Tax1 induces the expression of a wide range of host cell proteins, including transcription factors and cytokines such as IL-2, tumor necrosis factor (TNF), and others $(3,8)$. While Tax2 shares many properties that are characteristics of Tax1, the ability of Tax2 to induce cellular transformation and to induce cytokine gene expression have been reported to be lower than Tax1 (20). The differential functional and structural characteristics of Tax1 and Tax2 proteins may explain the differences in disease potential associated with HTLV-1 and HTLV-2 $(16,46)$. In clinical settings where overexpression of Tax1 occurs, increased transactivation of cellular genes might result in immune dysregulation in affected individuals (reviewed in 23).

The determinants of disease for human retroviruses are dictated in a significant way by the array of viral proteins that are responsible for regulating viral replication and the host cell response to infection. HTLV-1 has a region between the env gene and the 3- LTR that encodes for two regulatory proteins, Tax 1 and Rex1, which are produced from messages that are spliced differently from distinct overlapping reading frames. Rex 1 protein regulates the expression of viral messenger RNA (mRNA), and the Tax1 protein induces the expression of cell transcription factors that alter host cell gene expression (8). While it is clear that Tax1 and Tax2 each have a critical role in viral replication and host gene expression in the cellular microenvironment, less certain is whether these key viral accessory proteins are secreted and play a role in the extracellular environment. Extracellular detection of Tax1 has been shown in the serum and cerebrospinal fluid (CSF) of TSP/HAM patients (9). In addition, the release of Tax in the extracellular environment has also been reported from HTLV-1-infected cells (28). Extracellular Tax1 has been shown to induce cytokine production $(13,29)$; Tax 2 has been reported to be predominantly localized in the cytoplasm $(31,45)$, and detection of Tax2 in the extracellular pool has only been demonstrated in cell culture systems (45). A question raised by this study is whether the levels of chemokines detected during the in vitro assays would replicate the levels induced by HTLV-1 and HTLV-2 in vivo. Previous reports suggest that circulating levels of CC-chemokines can be detected in human plasma and CSF, and have been used as biomarkers to study inflammatory diseases $(2,14,42)$. Lewis et al. (25) previously documented the spontaneous production of CC-chemokines by individuals infected with HTLV-2 or with HIV-1/HTLV-2 co-infection, albeit at low levels; detectable levels of tax 2 mRNA were reported in lymphocytes of these donors $(6,25)$. Moreover, in vitro experiments showed that HTLV-2 Tax could increase 
A
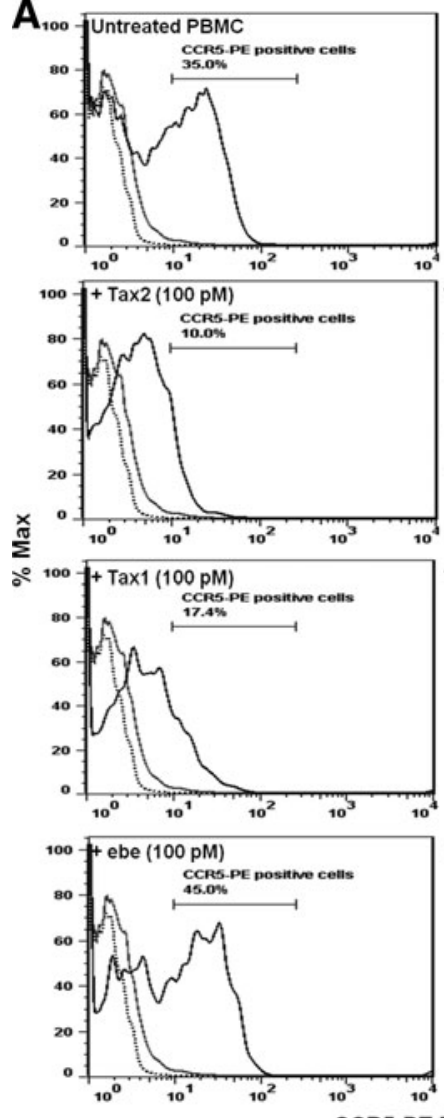
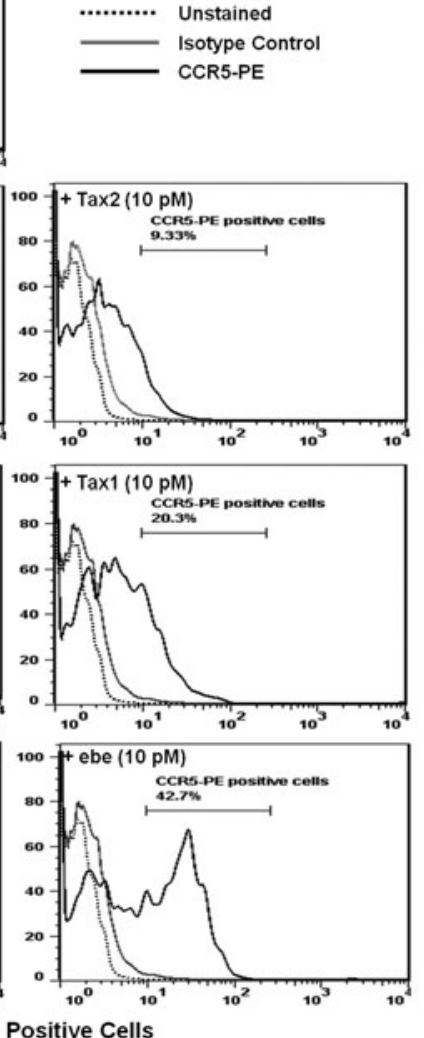

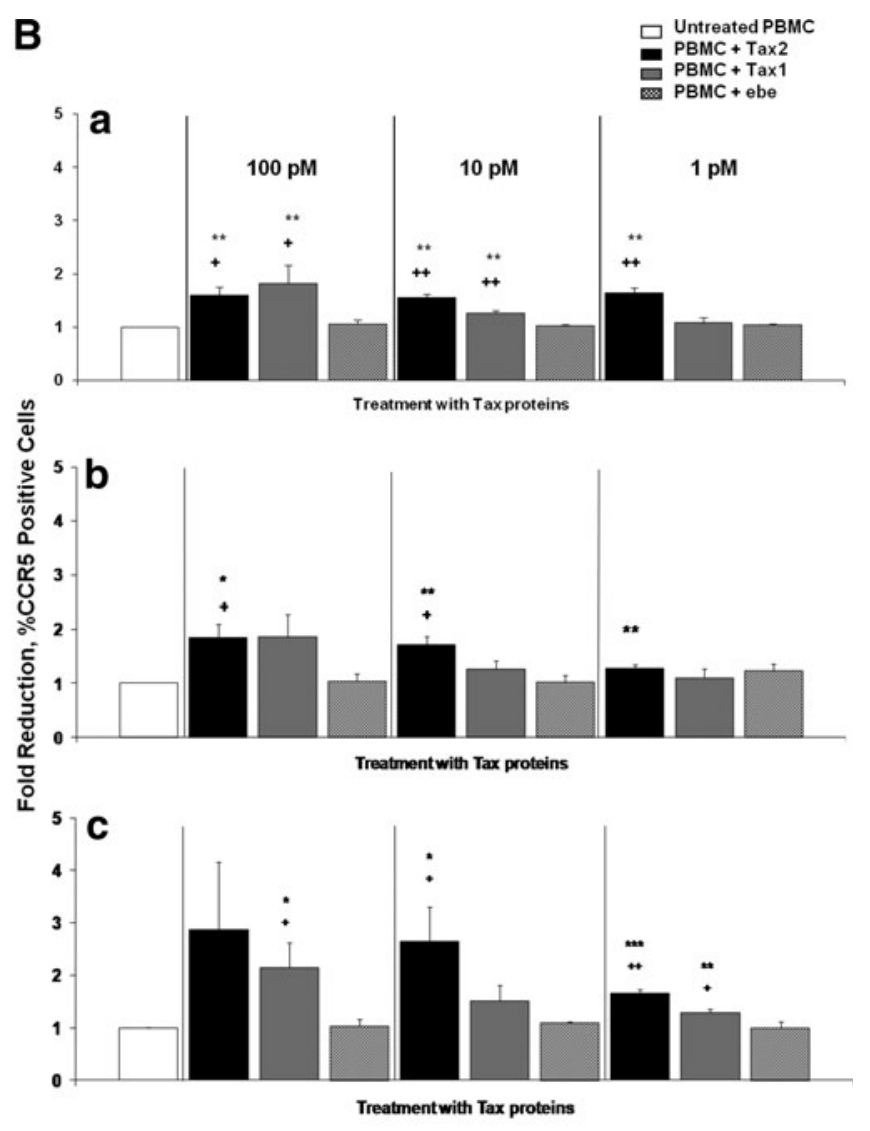

FIG. 5. Fold reduction of CCR5 receptor expression on lymphocytes from PBMCs treated with Tax protein. (A) Cell surface CCR5 expression on gated lymphocytes from PBMCs treated with Tax proteins are depicted in histograms showing the percentages of CCR5-PE-positive cells from PBMCs treated with 10 and $100 \mathrm{pM}$ of Tax1, Tax2, ebe as control, or untreated cells. Cells were stained either with a mixture of PE-conjugated CCR5 antibody (black lines), or isotype control (grey lines). Unstained cells are shown by the black dotted lines. (B) PBMCs treated with Tax proteins (10 and 100 pM) for (a) 24, (b) 48, and (c) $72 \mathrm{~h}$ were stained with CCR5-PE antibody, and analyzed by FACS. FlowJo software was used to determine percentages of CCR5-positive cells. Data are represented as mean \pm SEM of CCR5 fold reductions. Fold reductions of CCR5 were calculated as the percentage of CCR5-positive cells treated with Tax proteins or ebe control, divided by the percentage of untreated CCR5-positive cells $\left({ }^{*} p<0.05\right.$, ${ }^{* *} p<0.01$, and ${ }^{* *} p<0.001$ versus untreated; $+p<0.05$ and $++p<0.01$ versus ebe).

transcription from the MIP- $1 \beta$ and RANTES promoters in COS-7 cells (25). In addition, a high expression (a range of 30 - to 80 -fold) of RANTES and MIP-3 $\alpha$ /CCL20 was determined using an Ad-Tax1-transduced HeLa system (54). Here, both Tax 1 and Tax 2 proteins demonstrated induction of high levels of CC-chemokine secretion in human PBMCs. Of interest, in this study it was also demonstrated that significantly high levels of the spontaneous expression of RANTES was found in the supernatants of in vitro cultured non-activated PBMCs from HTLV-2-infected individuals. It may be conceivable that the elevated expression of RANTES may be due to Tax2, since this transactivator protein activates expression of numerous cellular genes. In fact, Mori et al. (33) found very high levels of RANTES/CCL5 in the culture supernatants of HTLV-1-infected T-cell lines and in PBMCs and lymph nodes from patients with acute- or chronic-type ATL. These patients were diagnosed based on clinical features, hematologic findings, and the presence of anti-HTLV-1 antibodies in patient sera. Monoclonal HTLV-1 provirus integration into the DNA of leukemic cells was confirmed by Southern blot hybridization (33).
The CC-chemokines are the natural ligands for CCR5, which is the essential co-receptor for HIV-1 cell entry $(5,15,34)$. Levels of protection against HIV-1 infection are multiple, including polymorphisms of the CCR5 chemokine receptor HLA B5701, the expression of particular KIR molecules on NK cells, and polymorphisms of loci on human chromosome 22, and also depend on the stimulation of both innate and acquired immunity (39). HIV-1-exposed individuals who remained uninfected produced high levels of CC-chemokines (38), thus suggesting that resistance to HIV-1 infection entails a complex network of immunologic interactions that take place in multiple anatomic sites $(38,39)$. Additionally, it has been shown that CC-chemokines suppress HIV-1 viral replication in vitro (11), which suggests a positive role for these molecules in controlling the natural course of HIV infection $(11,18)$. Several mechanisms have been postulated by which chemokines inhibit HIV-1 infection by mediating protective immunity. These include steric hindrance, chemokine receptor desensitization and internalization, and the formation of receptor dimers, structures no longer recognized by the virus (reviewed in 32). Downregulation of CCR5 expression was 
observed herein in non-activated HTLV-2-infected lymphocytes with concomitant expression of CCL5/RANTES. Furthermore, in this study we report that HTLV-2 Tax proteins displayed a potent ability to downregulate CCR5-receptor expression on lymphocytes, along with the induction of CC-chemokines. These findings support the hypothesis that Tax2 CC-chemokine induction could prevent interaction of the HIV-1env/CD4 complex to the CCR5 receptor, and may potentially hamper the virus from infecting the target cell. Therefore, Tax2 could play a key role in PBMC survival in patients with HIV-1/HTLV-2 co-infection.

In an observational cohort study of HIV-1/HTLV-1 and HIV-1/HTLV-2 co-infections, our group and others have reported that HIV-1/HTLV-2 co-infection was associated with improved survival, delayed rates of $\mathrm{CD}_{4}{ }^{+} \mathrm{T}$-cell decline, and a reduction in AIDS and AIDS-defining conditions (6,51). Both HTLV-1 and HTLV-2 viral loads were shown to be increased during retroviral co-infection, as shown by PCR detection of HTLV-1 and HTLV-2 tax mRNA and proviral DNA levels in PBMCs $(7,51)$. Levels of HTLV-1 tax mRNA and proviral DNA have been previously shown to correlate with disease severity among HTLV-1-infected carriers with TSP/HAM (53).

HTLV-2 infection has been associated with low proviral load (35); however, HTLV-1 and HTLV-2 tax mRNA levels among patients with HIV-1/HTLV-1 and HIV-1/HTLV-2 coinfections have been shown to be upregulated at levels similarly observed in patients with TSP/HAM (51). These observations lead to the possibility that Tax 1 and Tax2 could further dysregulate immune responses among HIV-1/ HTLV-1/-2 dually-infected individuals. It has been suggested that $\mathrm{CD}^{+}{ }^{+} \mathrm{T}$ cells of HTLV-2-infected individuals inhibited HIV-1 replication via secretion of CC-chemokines, particularly MIP- $1 \alpha$, since the levels were correlated with a high HTLV-2 proviral DNA load in vivo (40). Given these findings, it is possible that HTLV-1 and/or HTLV-2 may stimulate the production of CC-chemokines, directly or at a transcriptional level, via the viral transactivator Tax, thus altering the progression of HIV-1 infection in co-infected individuals (25).

\section{Conclusions}

Our study demonstrated a rapid and efficient uptake of Tax recombinant proteins by PBMCs at concentrations as low as $10 \mathrm{pM}$, which resulted in an induction of three CCchemokines and downregulation of CCR5 levels. These data may support the contention that HTLV-1 and HTLV-2 infections could result in stimulation of innate immunity through Tax1- and Tax2-mediated induction of CC-chemokines, thus preventing CCR5/HIV-1 binding $(11,12,25)$.

\section{Author Disclosure Statement}

No competing financial interests exist.

\section{Acknowledgments}

This work was supported by funds received from the National Institutes of Health-National Institute of Allergy and Infectious Diseases (R01 A149744), Advancing a Healthier Wisconsin endowment at the Medical College of Wisconsin, and a VA Merit Review grant (BX000488-01).

\section{References}

1. Alfano M, and Poli G: Role of cytokines and chemokines in the regulation of innate immunity and HIV infection. Mol Immunol 2005;42:161-182.

2. Armah HB, Wilson NO, Sarfo BY, et al.: Cerebrospinal fluid and serum biomarkers of cerebral malaria mortality in Ghanaian children. Malaria J 2007;6:147-163.

3. Banerjee P, Rochford R, Antel J, Canute G, Wrzesinski S, Sieburg M, and Feuer G: Proinflammatory cytokine gene induction by human T-cell leukemia virus type 1 (HTLV-1) and HTLV-2 Tax in primary human glial cells. J Virol 2007;81:1690-1700.

4. Bartman MT, Kaidarova Z, Hirschkorn D, et al.: Longterm increases in lymphocytes and platelets in human Tlymphotropic virus type II infection. Blood 2008;112:3995-4002.

5. Bates P: Chemokine receptors and HIV-1: an attractive pair? Cell 1996;86:1-3.

6. Beilke MA, Theall KP, O'Brien M, Clayton JL, Benjamin SM, Winsor EL, and Kissinger PJ: Clinical outcomes and disease progression among patients coinfected with HIV and human $\mathrm{T}$ lymphotropic virus types 1 and 2 . Clin Infect Dis 2004;39:2562-2563.

7. Beilke MA, Traina-Dorge VL, Sirois M, et al.: Relationship between human T lymphotropic virus (HTLV) type $1 / 2$ viral burden and clinical and treatment parameters among patients with HIV type 1 and HTLV-1/2 coinfection. Clin Infect Dis 2007;44:1229-1234.

8. Boxus M, Twizere JC, Legros S, Dewulf JF, Kettmann R, and Willems L: The HTLV-1 Tax interactome. Retrovirology 2008;5:76-100.

9. Cartier L, and Ramirez E: Presence of HTLV-I Tax protein in cerebrospinal fluid from HAM/TSP patients. Arch Virol 2005;150:743-753.

10. Chevalier SA, Meertens L, Pise-Masison C, et al.: The Tax protein from the primate T-cell lymphotropic virus type 3 is expressed in vivo and is functionally related to HTLV-1 Tax rather than HTLV-2 Tax. Oncogene 2006;25:4470-4482.

11. Cocchi F, DeVico AL, Garzino-Demo A, Arya SK, Gallo RC, and Lusso P: Identification of RANTES, MIP- $1 \alpha$, and MIP- $1 \beta$ as the major HIV-suppressive factor produced by CD8 $+\mathrm{T}$ cells. Science 1995;270:1811-1815.

12. Coffey MJ, Woffendin C, Phare SM, Strieter RM, and Markovitz DM: RANTES inhibits HIV-1 replication in human peripheral blood monocytes and alveolar macrophages. Am J Physiol Lung Cell Mol Physiol 1997;272:1025-1029.

13. Dhib-Jalbut S, Hoffman PM, Yamabe T, et al.: Extracellular human T-cell lymphotropic virus type I Tax protein induces cytokine production in adult human microglial cells. Ann Neurol 1994;36:787-790.

14. Domberg J, Liu C, Papewalis C, et al.: Circulating chemokines in patients with autoimmune thyroid diseases. Horm Metab Res 2008;40:416-421.

15. Dragic T, Litwin V, Allaway GP, et al.: HIV-1 entry into CD4 + cells is mediated by the chemokine receptor CC-CKR-5. Nature 1996;381:667-773.

16. Endo K, Hirata A, Iwai K, et al.: Human T-cell leukemia virus type 2 (HTLV-2) Tax protein transforms a rat fibroblast cell line but less efficiently than HTLV-1 Tax. J Virol 2002;76:2648-2653.

17. Gallo, R: Human retroviruses after 20 years: a perspective from the past and prospects their future control. Immunol Rev 2002;185:236-265.

18. Garzino-Demo A, Moss RB, Margolick JB, et al.: Spontaneous and antigen-induced production of HIV-inhibitory 
beta-chemokines are associated with AIDS-free status. Proc Natl Acad Sci USA 1999;96:11986-11991.

19. Grassmann R, Aboud M, and Jeang K-T: Molecular mechanisms of cellular transformation by HTLV-1 Tax. Oncogene 2005;24:5976-5985.

20. Higuchi M and Fujii M: Distinct functions of HTLV-1 Tax1 from HTLV-2 Tax2 contribute key roles to viral pathogenesis. Retrovirology 2009;6:117-128.

21. Hinuma Y, Nagata K, Hanaoka M, et al.: Adult T-cell leukemia: antigen in an ATL cell line and detection of antibodies to the antigen in human sera. Proc Natl Acad Sci USA 1981;78:6476-6480.

22. Kamoi K, Yamamoto K, Misawa A, et al.: SUV39H1 interacts with HTLV-1 Tax and abrogates Tax transactivation of HTLV-1 LTR. Retrovirology 2006;3:5.

23. Kannian P, Patrick L, and Green PL: Human T lymphotropic virus type 1 (HTLV-1): Molecular biology and oncogenesis. Viruses 2010;2:2037-2077.

24. Khurana S, Kennedy M, King LR, and Golding H: Identification of a linear peptide recognized by monoclonal antibody 2D7 capable of generating CCR5-specific antibodies with human immunodeficiency virus-neutralizing activity. J Virol 2005;79:6791-6800.

25. Lewis MJ, Gautier VW, Wang XP, Kaplan MH, and Hall WW: Spontaneous production of C-C chemokines by individuals infected with human $\mathrm{T}$ lymphotropic virus type II (HTLV-II) alone and HTLV-II/HIV-1 coinfected individuals. J Immunol 2000;165:4127-4132.

26. Lewis MJ, Sheehy N, Salemi M, VanDamme AM, and Hall WW: Comparison of CREB- and NF kappaB-mediated transactivation by human $\mathrm{T}$ lymphotropic virus type II (HTLV-II) and type I (HTLV-I) Tax proteins. Virology 2002;295:182-189.

27. Lindholm P, Marriott S, Gitlin S, and Brady JN: Differential precipitation and zinc chelate chromatography purification of biologically active HTLV-I Tax1 expressed in E. coli. J Biochem Biophys Methods 1991;22:233-241.

28. Lindholm, PF, Marriott SJ, Gitlin SD, Bohan CA, and Brady JN: Induction of nuclear NF-kappa B DNA binding activity after exposure of lymphoid cells to soluble tax1 protein. New Biol 1990;2:1034-1043.

29. Marriott SJ, Trinh, D, and Brady JN: Activation of interleukin-2 receptor alpha expression by extracellular HTLV-I Tax1 protein: a potential role in HTLV-I pathogenesis. Oncogene 1992;7:1749-1755.

30. Matsukawa A, Hogaboam CM, Lukacs NW, and Kunkel SL: Chemokines and innate immunity. Rev Immunogenet 2000;2:339-358.

31. Meerten L, Chevalier S, Weil R, Gessain A, and Mahieux R: A 10-amino acid domain within Human T-cell leukemia virus type 1 and type 2 Tax protein sequences is responsible for their divergent subcellular distribution. J Biol Chem Mol Biol 2004;279:43307-43320.

32. Mellado M, Rodríguez-Frade JM, Mañes S, and Martínez AC: Chemokine signaling and functional responses: the role of receptor dimerization and TK pathway activation. Annu Rev Immunol 2001;19:397-421.

33. Mori N, Krensky AM, Ohshima K, et al.: Elevated expression of CCL5/RANTES in adult T-cell leukemia cells: possible transactivation of the CCL5 gene by human T-cell leukemia virus type I tax. Int J Cancer 2004;111:548-557.

34. Murdoch $C$ and Finn A: Chemokine receptors and the role in inflammation and infectious disease. Blood 2000;95:30323043.
35. Murphy EL, Lee T-H, Chafets D, et al.: Higher human $\mathrm{T}$ lymphotropic virus (HTLV) provirus load is associates with HTLV-1 versus HTLV-II, with HTLV-II subtype A versus B, and with male sex and a history of blood transfusion. J Infect Dis 2004;190:504-510.

36. Newbound GC, Andrews JM, O'Rourke JP, Brady JN, and Lairmore MD: Human T-cell lymphotropic virus type 1 Tax mediates enhanced transcription in CD4 + T lymphocytes. J Virol 1996;70:2101-2106.

37. Osame M, Usuku K, Izumo S, et al:: HTLV-I associated myelopathy, a new entity. Lancet 1986;1:1031-1032.

38. Paxton WA, Martin SR, Tse D, et al.: Relative resistance to HIV-1 infection of CD4 lymphocytes from persons who remain uninfected despite multiple high-risk sexual exposures. Nat Med 1996;2:412-417.

39. Piacentini L, Fenizia C, Naddeo V, and Clerici M: Not just sheer luck! Immune correlates of protection against HIV-1 infection. Vaccine 2008;26:3002-3007.

40. Pilotti E, Elviri L, Vicenzi E, et al.: Postgenomic up-regulation of CCL3L1 expression in HTLV-2-infected persons curtails HIV-1 replication. Blood 2007;109:1850-1856.

41. Poiesz BJ, Ruscetti FW, Gazdar AF, Bunn PA, Minna JD, and Gallo RC: Detection and isolation of type $C$ retrovirus particles from fresh and cultured lymphocytes of a patient with cutaneous T-cell lymphoma. Proc Natl Acad Sci USA 1980;77:7415-7419.

42. Pokkali S and Das SD: Expression of CXC and CC type of chemokines and its receptors in tuberculous and non-tuberculous effusions. Cytokine 2008;41:307-314.

43. Rodgers-Johnson P, Gajdusek DC, Morgan OS, Zaninovic V, Sarin PS, and Graham DS: HTLV-I and HTLV-III antibodies and tropical spastic paraparesis. Lancet 1985;2:1247-1248.

44. Semmes OJ, Majone F, Cantemir C, Turchetto L, Hjelle B, and Jeang KT: HTLV-I and HTLV-II Tax: differences in induction of micronuclei in cells and transcriptional activation of viral LTRs. Virology 1996;217:373-379.

45. Sieburg M, Tripp A, Ma JW, and Feuer G: Human T-cell leukemia virus type 1 (HTLV-1) and HTLV-2 Tax oncoproteins modulate cell cycle progression and apoptosis. J Virol 2004;78:10399-10409.

46. Shoji T, Higuchi M, Kondo R, et al.: Identification of a novel motif responsible for the distinctive transforming activity of human T-cell leukemia virus (HTLV) type 1 Tax1 protein from HTLV-2 Tax2. Retrovirology 2009;6:83.

47. Shuh $X$ and Beilke M: The human T-cell leukemia virus type 1 (HTLV-1): new insights into the clinical aspects and molecular pathogenesis of adult T-cell leukemia/lymphoma (ATLL) and tropical spastic paraparesis/HTLV-associated myelopathy (TSP/HAM). Microsc Res Tech 2005;68:176-196.

48. Sowa JM: Human T lymphotropic virus I, myelopathy, polymyositis and synovitis: an expanding rheumatic spectrum. J Rheumatol 1992;19:316-318.

49. Tanaka Y, Hayashi M, Takagi S, and Yoshie O: Differential transactivation of the intercellular adhesion molecule 1 gene promoter by Tax1 and Tax2 of human T-cell leukemia viruses. J Virol 1996;70:8508-8517.

50. Tang Y, Tie F, Boros I, Harrod R, Glover M, and Giam C-Z: An extended $\alpha$-helix and specific amino acid residues opposite the DNA-binding surface of the cAMP response element binding protein basic domain are important for human $\mathrm{T}$ cell lymphotropic retrovirus type I Tax binding. J Biol Chem 1998;273:27339-27346.

51. Turci M, Pilotti E, Ronzi P, et al.: Coinfection with HIV-1 and human T-cell lymphotropic virus type II in intravenous drug 
users is associated with delayed progression to AIDS. J Acquir Immune Defic Syndr 2006;41:100-106.

52. Xie L, Yamamoto B, Haoudi A, Semmes OJ, and Green PL: PDZ binding motif of HTLV-1 Tax promotes virus-mediated T-cell proliferation in vitro and persistence in vivo. Blood 2006;107:1980-1988.

53. Yamano $\mathrm{Y}$, Nagai M, Brennan M, et al.: Correlation of human T-cell lymphotropic virus type 1 (HTLV-1) mRNA with proviral DNA load, virus-specific CD8 $(+)$ T cells, and disease severity in HTLV-1-associated myelopathy (HAM/ TSP). Blood 2002;99:88-94.

54. Yang L, Kotomura N, Ho YK, Zhi H, Bixler S, Schell MJ, and Giam CZ: Complex cell cycle abnormalities caused by human T-lymphotropic virus type 1 Tax. J Virol 2011;85:30013009.

55. Yoshida M, Seiki M, Yamaguchi K, and Takatsuki K: Monoclonal integration of human T-cell leukemia provirus in all primary tumors of adult T-cell leukemia suggests causative role of human T-cell leukemia virus in the disease. Proc Natl Acad Sci USA 1984;81:2534-2537.

\author{
Address correspondence to: \\ Dr. Mark A. Beilke \\ Infectious Diseases Division \\ Department of Medicine \\ Medical College of Wisconsin \\ 9200 W. Wisconsin Avenue \\ Milwaukee, WI 53226 \\ E-mail: mbeilke@mcw.edu
}

Received May 25, 2011; accepted July 24, 2011. 
